\title{
THE DYNAMICS OF SCALAR-TENSOR COSMOLOGY FROM RS TWO-BRANE MODEL
}

\author{
P. KUUSK*, L. JÄRV, and M. SAAL \\ Institute of Physics, University of Tartu, \\ Riia 142, 51014, Tartu, Estonia \\ *E-mail: piret.kuusk@ut.ee
}

\begin{abstract}
We consider Randall-Sundrum two-brane cosmological model in the low energy gradient expansion approximation by Kanno and Soda. It is a scalar-tensor theory with a specific coupling function. We find a first integral of equations for the A-brane metric and estimate constraints for the dark radiation term. We perform a complementary analysis of the dynamics of the scalar field (radion) using phase space methods and examine convergence towards the limit of general relativity. We find that it is possible to stabilize the radion at a finite value with suitable negative matter densities on the B-brane.
\end{abstract}

Keywords: two-brane cosmology, scalar-tensor theory in the Jordan frame, phase space methods.

\section{Introduction}

Following Kanno and Soda, ${ }^{1}$ we consider the Randall-Sundrum type I cosmological scenario $^{2}$ with two branes (A and B) moving in a 5-dimensional bulk. Both branes are taken to be homogeneous and isotropic and supporting energy-momentum tensors of a perfect barotropic fluid with barotropic index $\Gamma(p=(\Gamma-1) \rho)$ on the A-brane (identified with our visible Universe) and $\Gamma^{B}$ on the B-brane; for simplicity we assume $\Gamma=\Gamma^{B}$.

The field equations of the effective 4-dimensional theory obtained by Kanno and Soda ${ }^{1}$ are the equations of a scalar-tensor theory with one scalar field $\Psi$ (interpreted as a radion) with a specific coupling function $\omega(\Psi)=3 \Psi /(2(1-\Psi))$ which describes the proper distance between the branes. The cosmology of this model was first studied by Kanno et al. ${ }^{3}$ and later by us. ${ }^{4,5}$

\section{Field equations and analytic solutions}

The field equations ${ }^{1}$ on the A-brane for the Friedmann-Lemaitre-Robertson-Walker (FLRW) line element $d s^{2}=-d t^{2}+a^{2}(t)\left[\left(1-k r^{2}\right)^{-1} d r^{2}+r^{2} d \Omega^{2}\right]$ and perfect fluid matter on both branes read $(H \equiv \dot{a} / a)$

$$
\begin{array}{r}
H^{2}=-H \frac{\dot{\Psi}}{\Psi}+\frac{1}{4} \frac{\dot{\Psi}^{2}}{\Psi(1-\Psi)}+\frac{\kappa^{2}}{3} \frac{V}{\Psi}+\frac{\kappa^{2}}{3} \frac{\rho}{\Psi}+\frac{\kappa^{2}}{3} \frac{(1-\Psi)^{2}}{\Psi} \rho^{B}-\frac{k}{a^{2}},(1) \\
2 \dot{H}+3 H^{2}=-\frac{\kappa^{2}}{\Psi} p-\frac{\kappa^{2}}{\Psi}(1-\Psi)^{2} p^{B}+\frac{\kappa^{2}}{\Psi} V-\frac{\ddot{\Psi}}{\Psi}-2 H \frac{\dot{\Psi}}{\Psi}-\frac{3}{4} \frac{\dot{\Psi}^{2}}{\Psi(1-\Psi)}-\frac{k}{a^{2}},(2)
\end{array}
$$




$$
\begin{aligned}
\ddot{\Psi}= & -3 H \dot{\Psi}-\frac{1}{2} \frac{\dot{\Psi}^{2}}{(1-\Psi)}+\frac{2}{3} \kappa^{2}\left(2 V-\Psi \frac{d V}{d \Psi}\right)(1-\Psi) \\
& +\frac{\kappa^{2}}{3}(1-\Psi)(\rho-3 p)+\frac{\kappa^{2}}{3}(1-\Psi)^{2}\left(\rho^{B}-3 p^{B}\right),
\end{aligned}
$$

they reduce to general relativity when $\Psi \rightarrow 1, \dot{\Psi} \rightarrow 0$. The conservation laws as measured by an A-brane observer, $\dot{\rho}+3 H \Gamma \rho=0, \dot{\rho}^{B}+3 H \Gamma \rho^{B}-\left(3 \dot{\Psi} \Gamma \rho^{B}\right) /(2(1-$ $\Psi))=0$, imply a relation between the energy densities on the A- and B-brane

$$
\rho^{B}=\rho_{0}^{B}\left(\frac{1-\Psi}{1-\Psi_{0}}\right)^{-\frac{3}{2} \Gamma}\left(\frac{\rho}{\rho_{0}}\right)
$$

The dynamical equation for $H$ decouples from the scalar field and B-brane matter ${ }^{4,5}$ due to the specific form of the coupling function $\omega(\Psi)$ and its first integral reads

$$
H^{2}=\frac{\kappa^{2}}{3} \sigma+\frac{\kappa^{2}}{3} \rho_{0}\left(\frac{a}{a_{0}}\right)^{-3 \Gamma}-\frac{k}{a^{2}}+\frac{\kappa^{2} C}{3}\left(\frac{a}{a_{0}}\right)^{-4},
$$

which is a Friedmann equation with a dark radiation term, $C$. Comparison with recent results of light element abundances, $\mathrm{BBN}$, and $\mathrm{CMB}$ observations constrain the dark radiation term ${ }^{5}$ ( $\rho_{0}$ is the radiation energy density),

$$
-0.054 \leq \frac{C}{\rho_{0}} \leq 0.138 \text {. }
$$

Analytic solutions are found ${ }^{5}$ for the flat Universe scale factor in the case of cosmological constant $(\Gamma=0)$, radiation $(\Gamma=4 / 3)$, dust $(\Gamma=1)$, cosmological constant and radiation, and for the scalar field in the case of radiation $(\Gamma=4 / 3)$, cosmological constant and radiation (to be expressed in terms of elliptic functions).

\section{The dynamics of the scalar field}

Defining a new time variable ${ }^{6}, d p=h_{c} d t, \quad h_{c}=H+\frac{\dot{\Psi}}{2 \Psi}$, it is possible to derive a decoupled "master equation" for the scalar field (here $V=0, k=0, \frac{d f}{d p} \equiv f^{\prime}$ ),

$$
\begin{aligned}
8(1-\Psi) \frac{\Psi^{\prime \prime}}{\Psi} & -3(2-\Gamma)\left(\frac{\Psi^{\prime}}{\Psi}\right)^{3}-2((4-6 \Psi)-(4-3 \Gamma)(1-\Psi) W(\Psi))\left(\frac{\Psi^{\prime}}{\Psi}\right)^{2} \\
& +12(2-\Gamma)(1-\Psi) \frac{\Psi^{\prime}}{\Psi}-8(4-3 \Gamma)(1-\Psi)^{2} W(\Psi)=0
\end{aligned}
$$

where

$$
W(\Psi)=\frac{1+(1-\Psi) \frac{\rho_{0}^{B}}{\rho_{0}}\left(\frac{1-\Psi}{1-\Psi_{0}}\right)^{-\frac{3}{2} \Gamma}}{1+(1-\Psi)^{2} \frac{\rho_{0}^{B}}{\rho_{0}}\left(\frac{1-\Psi}{1-\Psi_{0}}\right)^{-\frac{3}{2} \Gamma}} \quad \text { or } \quad W(\Psi)=\frac{1+(1-\Psi) \frac{\sigma \mathrm{B}}{\sigma}}{1+(1-\Psi)^{2} \frac{\sigma \mathrm{B}}{\sigma}}
$$

corresponding to the case when $\sigma=0, \sigma^{B}=0, \rho \neq 0, \rho^{B} \neq 0$ and to the case when $\sigma \neq 0, \sigma^{B} \neq 0, \rho=0, \rho^{B}=0$, respectively. Phase portraits are found ${ }^{5}$ depending on the values of constraints involved (Fig. 10). 


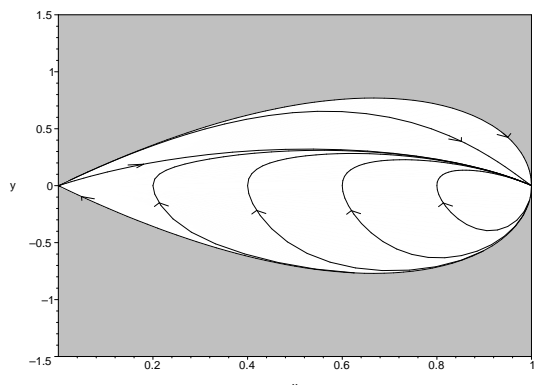

(a)

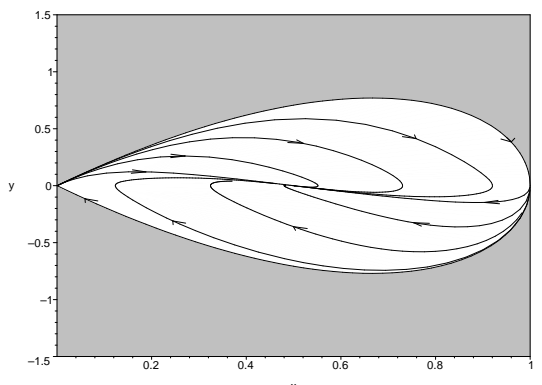

(b)

Fig. 1. Phase portraits $\left(x=\Psi(p), y=\Psi^{\prime}(p)\right)$ (a) for cosmological constants: $\sigma=1, \sigma^{B}=-0.5$ and (b) for dust: $\rho_{0}=0.5, \rho_{0}^{B}=-1, \Psi_{0}=0.5$.

All trajectories are constrained to be in the physically allowed region of the phase space determined by Friedmann equation (11). Figure 1a for a cosmological constant dominated Universe contains a saddle point $\left(\Psi=0, \Psi^{\prime}=0\right)$ and a spiralling attractor corresponding to general relativity $\left(\Psi=1, \Psi^{\prime}=0\right)$. Figure $1 \mathrm{~b}$ for a dust dominated Universe with $\rho_{0}^{B}<0$ contains two saddle points, $\left(\Psi=0, \Psi^{\prime}=0\right)$ and $\left(\Psi=1, \Psi^{\prime}=0\right)$, and also an attractor $\left(\Psi=1-\rho_{0}^{B}{ }^{2}(1-\Psi)^{3} / \rho_{0}^{2}, \Psi^{\prime}=0\right)$ which can stabilize the branes in a position that does not correspond to general relativity on the A-brane.

\section{Summary}

We have considered a braneworld inspired scalar-tensor cosmology with a specific coupling function, cosmological constant and perfect fluid matter on both branes. The first integral of equations for the metric tensor of the A-brane contains the dark radiation term. Phase portraits of the scalar field reveal fixed points and allow us to find late time fates for different cosmological models. The solutions may approach general relativity $(\Psi=1)$, or go to brane collision $(\Psi=0)$, depending on the initial conditions. There are also additional fixed points in between the two extremes: a saddle for cosmological constants and an attractor for dust (Fig. 1(b)).

\section{References}

1. S. Kanno and J. Soda, Phys. Rev. D 66, 083506 (2002), hep-th/0207029].

2. L. Randall and R. Sundrum, Phys. Rev. Lett. 83, 3370 (1999), hep-ph/9905221.

3. S. Kanno, M. Sasaki, and J. Soda, Prog. Theor. Phys. 109, 357 (2003), hep-th/0210250.

4. P. Kuusk and M. Saal, Gen. Rel. Grav. 36, 1001 (2004), gr-qc/0309084.

5. L. Järv, P. Kuusk, and M. Saal, Phys. Rev. D 75, 023505 (2007), gr-qc/0608109.

6. T. Damour and K. Nordtvedt, Phys Rev. D 48, 3436 (1993). 